\title{
Multiscale-Multiphysics Models of Ventricular Electromechanics - Computational Modeling, Parametrization and Experimental Validation
}

\author{
G. Plank ${ }^{1}$, A.J. Prassl ${ }^{1}$, R. Arnold ${ }^{1}$, Y. Rezk ${ }^{1}$, T.E. Fastl ${ }^{1}$, E. Hofer ${ }^{1}$ and C.M. Augustin ${ }^{1}$ \\ ${ }^{1}$ Medical University of Graz/Institute of Biophysics, Graz, Austria
}

\begin{abstract}
Computational modeling of ventricular electromechanics is considered to be among the most promising approaches to gain novel insight into cardiac function in health and disease. Such models allow to integrate the wealth of available experimental data into a mechanistic framework which allows to study complex cause-effect relationships across several scales of biological organization - ranging from subcellular processes such as cellular force generation up to the organ scale. However, due to the enormous challenges involved in constructing such models, the number of available multiscale models is very limited. Their implementation resorts to very crude approximations which ignores many aspects of known biophysical details and/or uses extremely coarse spatio-temporal discretizations to reduce computational complexity.

In this study we focus on a subset of the parametrization and validation issues in models of cardiac electromechanics. An experimental setup for measuring passive stress-strain relationships as well as isometric force transients in thin preparations of ventricular trabeculae or papillary muscles was developed along with a matching computer model to parametrize and validate passive and active mechanical model responses at the tissue scale.
\end{abstract}

Keywords- Cardiac electrophysiology, Cardiac electromechanics, Bidomain model.

\section{INTRODUCTION}

Computational modeling of ventricular electromechanics is considered to be among the most promising approaches to gain novel insight into cardiac function in health and disease. Such models allow to integrate the wealth of available experimental data into a mechanistic framework which facilitates studying complex cause-effect relationships across several scales of biological organization - ranging from subcellular processes up to the organ scale. However, due to the enormous multi-faceted challenges involved in constructing such models, the number of available multiscale-multiphysics frameworks is limited. Moreover, most implementations resort to rather crude approximations which ignore many known biophysical details and employ fairly coarse spatio-temporal discretizations to reduce computational complexity.

Conceptually, the problem of building multiscale organ models of cardiac function is tackled by assembling basic building blocks which characterize different functional aspects and length scales. A fundamental component are models of cellular dynamics which describe electrophysiological (EP) behavior. Numerous models specialized for different species, tissues and pathologies have been reported in the literature over the past few decades. Most of them predict intracellular calcium transients, the main mediator signal of cellular force generation, thus allowing to couple the electrical excitation and recovery process to mechanical contraction and relaxation, a phenomenon referred to as excitation contraction coupling (ECC). Various models describing cellular force generation based on sliding filament theory exist [1] which can be coupled to EP models.

At the tissue and organ scale, the cardiac bidomain equations are the most relevant model for describing tissue responses to stimuli and the spread of activation and repolarization throughout the organ. The subjectspecific parametrization of the bidomain equations remains to be a challenging and unresolved problem. Only a limited number of experimental reports on key parameters such as tissue conductivities exist which are afflicted with a large margin of error [2]. Further, there is a significant inter-individual variation in the electrical activation sequence, as evidenced by variations in electrocardiogram waveform recorded from the body surface of individuals, due to factors such as topological variability of the specialized conduction system [3], variations in spatial heterogeneity of tissue structure [4], and EP heterogeneities which profoundly influence repolarization sequences [5]. During the contraction phase ac- 
tive forces have to act against the passive mechanical stiffness of myocytes which are embedded in the extracellular matrix and reinforced by the collagen fiber fabric at the tissue scale. These passive mechanical properties are investigated by multi-axial stretch experiments to parametrize constitutive relations [6] which are incorporated in the continuum mechanics framework. Finally, at the organ scale additional mechanical loads are imposed by time-varying pressures acting in the cavities against the endocardium. These pressures are typically accounted for by lumped parameter models of the circulatory system which are coupled as inhomogeneous Neumann type boundary conditions.

While all these models are carefully validated at the individual length scales and experimental conditions they were designed for, their integration into a comprehensive organ model leads to inconsistencies due to the numerous differences in terms of species, temperature, experimental protocol, etc. In general, one cannot expect models based on the direct assembly of these sub-models to yield a sufficiently close match with experimental observations at the organ scale. Rather, significant deviations at the organ scale are common, requiring a complex reparametrization of basic model parameters to achieve a better match with the organ's global response. Unlike in recent large-scale studies where it was attempted to parametrize in silico models of ventricular electromechanics in a patient-specific fashion at the organ scale, in this study we report on our efforts aimed at building an experimental setup suitable for measuring passive strain-stress relationships and active force transients under isometric conditions in thin strands of ventricular tissue, such as trabeculae or small papillary muscles. The limited geometric complexity of such preparations facilitates a quick iteration between experimental recordings and computer simulations to achieve a very close match between experimental observation and model prediction.

\section{Methods}

Modeling of cardiac electromechanics relies upon discretizations of the bidomain equations to characterize electrophysiology at the tissue scale, and the finite deformation elasticity equations to describe cardiac deformation under mechanical loads and intrinsic active force generation and relaxation. In a strongly coupled multiphysics scenario both sets of equations have to be solved together, necessitating the bidirectional exchange of data between the two physics. Hence, the two physics are bidirectionally coupled. Electrical activation triggers ECC - generated stresses influence the state of deformation and mechanical deformation influences upon the electrical tissue state via mechanoelectric feedback (MEF) - mechanical stresses or strains influence upon cellular physiology via different mechanisms such as length dependent tension, calcium sensitivity or stretch-activated channels.

\section{A. Experimental Model}

From an experimental point of view, a rigorous validation of an electromechanical model of a specific preparation across several scales of biological organization ranging from the subcellular to the organ scale - is not feasible. This is due to the large number of variables in such models which cannot be measured at a sufficiently high spatio-temporal resolution at the same time. Thus, to assess the validity of computational models of ventricular electromechanics a reduction of complexity is indispensable. For this sake, a simplified experimental setup for measuring force transients in preparations of ventricular trabeculae was developed. Analog to pressure-volume relationships such as measured in passive inflation experiments at the ventricular level, this setup allows to measure passive stress-strain relationships to parametrize passive constitutive equations as well as active force transients under isometric conditions, as elicited by electrical stimulation.

A

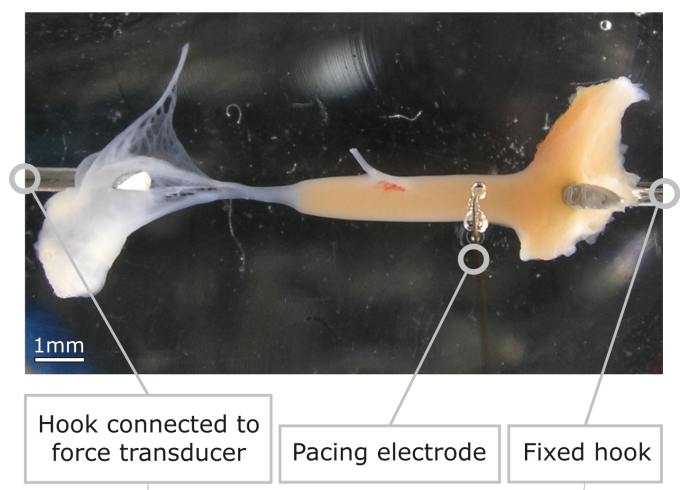

B

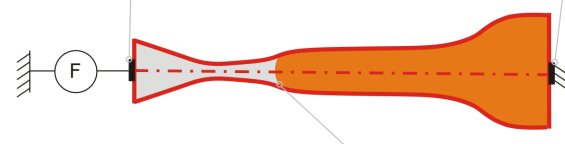

Muscle-tendon boundary

Fig. 1: A: Experimental setup for measuring force transients in small muscle preparations. B: Cross section of a computer model built to match the geometry of the preparation shown in $\mathrm{A}$. 
The experimental setup is shown in Fig. 1A. Rabbit ventricular trabeculae and papillary muscles were excised and stored in cold cardioplegic Tyrode's solution containing (in mM): $\mathrm{Na}^{+} 104, \mathrm{KCl} 5.4, \mathrm{NaHCO}_{3}$ 24, $\mathrm{MgCl}_{2} 1.15, \mathrm{NaH}_{2} \mathrm{PO}_{4} 0.42, \mathrm{CaCl}_{2}$ 0.2, glucose 5.6, $\mathrm{C}_{8} \mathrm{H}_{18} \mathrm{~N}_{2} \mathrm{O}_{4} \mathrm{~S}$ (HEPES) 10, and 2,3-butanedionemonoxime (BDM) 30, equilibrated with $95 \% \mathrm{O}_{2}$ and $5 \%$ $\mathrm{CO}_{2}$ to a $\mathrm{pH}$ of 7.4 .

The muscle stripes were transferred to a temperature controlled organ bath at $36^{\circ} \mathrm{C}$ and superfused with oxygenated Tyrode's solution containing (in $\mathrm{mM}$ ): $\mathrm{Na}^{+} 132, \mathrm{KCl} 5.4, \mathrm{NaHCO}_{3} 24, \mathrm{MgCl}_{2}$ 1.15, $\mathrm{NaH}_{2} \mathrm{PO}_{4}$ 0.42, $\mathrm{CaCl}_{2} 2.5$, glucose 5.6, $\mathrm{C}_{8} \mathrm{H}_{18} \mathrm{~N}_{2} \mathrm{O}_{4} \mathrm{~S}$ (HEPES) 10 , $\mathrm{pH}$ 7.4. Preparations were paced at a constant cycle length of $1000 \mathrm{~ms}$. Length changes $\Delta L$ were applied in steps of $50-200 \mu \mathrm{m}$, increasing the stretch ratio $\lambda=$ $\left(L_{0}+\Delta L\right) / L_{0}$ from 1 to 1.2 . Length $L$ was kept constant for 15-40 min after each step change until active force transients reached steady state. Maximum magnitudes of force transients and diastolic forces, measured as the baseline force in between active force twitches, were recorded to facilitate the construction of force-stretch ratio diagrams (Fig. 2).

\section{B. Governing Equations}

The set of equations governing cardiac electromechanics is given by

$$
\begin{aligned}
\nabla \cdot\left(\boldsymbol{\sigma}_{\mathrm{i}}+\boldsymbol{\sigma}_{\mathrm{e}}\right) \mathbf{C}^{-1} \nabla \phi_{\mathrm{e}} & =-\nabla \cdot \boldsymbol{\sigma}_{\mathrm{i}} \mathbf{C}^{-1} \nabla V_{\mathrm{m}}, \\
\nabla \cdot \boldsymbol{\sigma}_{\mathrm{i}} \mathbf{C}^{-1} \nabla V_{\mathrm{m}} & =-\nabla \cdot \boldsymbol{\sigma}_{\mathrm{i}} \mathbf{C}^{-1} \nabla \phi_{\mathrm{e}}+\beta I_{\mathrm{m}}, \\
I_{\mathrm{m}} & =C_{\mathrm{m}} \frac{\partial V_{\mathrm{m}}}{\partial t}+I_{\mathrm{ion}}\left(V_{\mathrm{m}}, \boldsymbol{\eta}\right), \\
V_{\mathrm{m}} & =\phi_{\mathrm{i}}-\phi_{\mathrm{e}}, \\
\frac{\partial \boldsymbol{\eta}}{\partial t} & =f\left(\boldsymbol{\eta}, V_{\mathrm{m}}, \boldsymbol{\sigma}_{\mathrm{a}}\right), \\
-\operatorname{div} \boldsymbol{\sigma}(\mathbf{u}) & =\mathbf{0}, \\
\boldsymbol{\sigma} & =\boldsymbol{\sigma}_{\mathrm{p}}+\boldsymbol{\sigma}_{\mathrm{a}}, \\
\boldsymbol{\sigma}_{\mathrm{p}} & =2 J^{-1} \mathbf{F} \frac{\partial \Psi(\mathbf{C})}{\partial \mathbf{C}} \mathbf{F}^{\mathrm{T}}, \\
\boldsymbol{\sigma}_{\mathrm{a}} & =\sigma_{\mathrm{a}}(\hat{\mathbf{f}} \otimes \hat{\mathbf{f}}), \\
\sigma_{\mathrm{a}} & =h\left(V_{\mathrm{m}}, \boldsymbol{\eta}, \lambda, \dot{\lambda}\right) .
\end{aligned}
$$

In the bidomain equations (1)-(4) describing electrophysiology, $\phi_{\mathrm{i}}$ and $\phi_{\mathrm{e}}$ are the intracellular and extracellular potentials, respectively, $V_{\mathrm{m}}=\phi_{\mathrm{i}}-\phi_{\mathrm{e}}$ is the transmembrane voltage, $\boldsymbol{\sigma}_{\mathrm{i}}$ and $\boldsymbol{\sigma}_{\mathrm{e}}$ are the intracellular and extracellular conductivity tensors, respectively, $\beta$ is the membrane surface to volume ratio, $I_{\mathrm{m}}$ is the transmembrane current density, $C_{\mathrm{m}}$ is the membrane capacitance per unit area, and $I_{\text {ion }}$ is the membrane ionic current density which depends on $V_{\mathrm{m}}$ and a set of state variables, $\boldsymbol{\eta}$. Deformation is governed by (5)-(8) with (8) linking electrics and mechanics.

The deformation is governed by the equilibrium equation (5) with unknown displacement field $\mathbf{u}$. The stress tensor $\boldsymbol{\sigma}$ consists of a passive (7) and an active contribution (8), while the latter depends on the normalized myocyte orientation $\hat{\mathbf{f}}$. Here, $\mathbf{F}$ is the deformation gradient, $\mathbf{C}=\mathbf{F}^{\mathrm{T}} \mathbf{F}$ is the right Cauchy-Green tensor, and $\Psi$ is a function which describes the strain energy density of the specific material. Using a particular cell model, indicated by the function $h(\bullet)$, the scalar-valued active stress term $\sigma_{\mathrm{a}}$ is computed (9), thus providing the link for ECC. To specify prescribed displacements or tractions, e.g., pressure, additional boundary conditions are incorporated.

\section{Discretization and Numerical Solution}

Contours of experimental preparations were manually extracted and discretized. Assuming rotational symmetry virtual 3D image stacks were generated. Image stacks were fed into the image-based unstructured mesh generation software Tarantula (CAE Software Solutions, Eggenburg, Austria) which builds fully unstructured, boundary fitted, locally refined, hex-dominant hybrid tessellations [7]. Subsequently, the hybrid mesh was tessellated into tetrahedral elements and each finite element was tagged either as electrically and mechanically active myocardium or as electrically and mechanically passive connective tissue (Fig. 1B).

Spatial discretization relied upon the finite element (FE) method where linear tetrahedral elements were used for both electrical and mechanical grid to discretize a strand model of a thin papillary muscle (diameter $\approx 0.9 \mathrm{~mm}$ ). Homogeneous Dirichlet boundary conditions were applied at the root of the muscle as well as at the distal end of the muscle where tendons, modeled as a passive nonlinear material, were also fixed, impeding displacement there in any direction (Fig. 1B). The same FE grid was used for both problems, thus facilitating an easier exchange of data in the multiphysics problem. The Mahajan-Shiferaw model of a rabbit ventricular myocyte [8] coupled to an active stress model [9] was employed to describe ECC. MEF remained unaccounted for at this preliminary stage of the model development. Data between electrical grid where quantities are represented 
at the nodes spanning the elements and the integration points of the mechanics grid were transferred by interpolation using FE shape functions. An electrical stimulus was applied at the proximal end of the papillary muscle to initiate propagation and mechanical contraction. Under the given boundary conditions contractions were isometric, however, inner shortening of sarcomeres was possible due to the compliance of the tendons and the dynamics of the contraction process which led to prestretching of later activating regions of the muscle. Standard iterative solvers were used for solving the bidomain equations [10]. A custom-tailored unsmoothed aggregation algebraic multigrid preconditioner for an iterative conjugate gradient solver was used to solve the equilibrium equation (5).

\section{RESULTS}

A set of 8 experiments on rabbit trabeculae and papillary muscles was performed. Both baseline passive forces as well as magnitudes of active force transients were recorded to construct force-stretch ratio diagrams. A sample result of one preparation is shown in Fig. 2. Further experimental results are in the process of being analyzed to provide a reference against which computer simulations can be gauged.

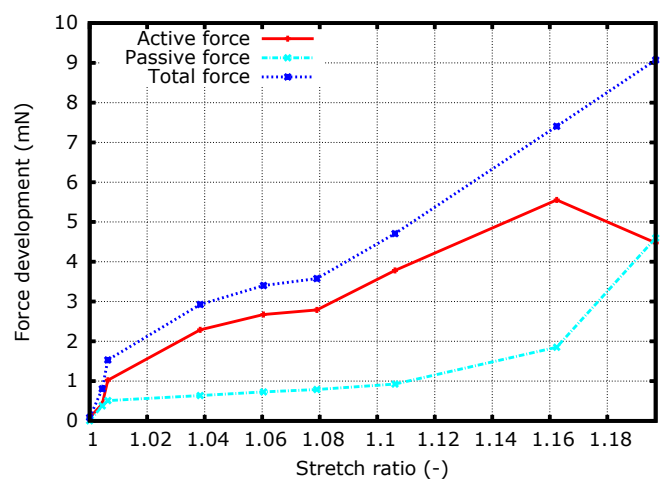

Fig. 2: Example of measured force magnitudes as a function of stretch ratio.

\section{Discussion}

In this study we describe the development of a basic experimental setup for measuring passive forces and active force transients under isometric conditions in thin strand-like ventricular tissue preparations. So far only a small number of experiments has been performed to provide a reference dataset against which a matching computer model can be validated. More experiments have to be conducted to better quantify the variability in both passive and active mechanical properties. A computer model matching one particular setup has been built using generic model parameters taken from the literature. The Mahajan-Shiferaw model was weakly coupled to the Rice active stress model in a coupled electromechanical tissue scale simulation setup. In a next step the computer model will be modified to allow the replication of the experimental protocol. All available datasets will be analyzed to quantify the range of variability in these experiments. These data will serve to steer the further development of the computer model to achieve a close match between experimental observation and model prediction.

\section{ACKNOWLEDGMents}

This research is supported by a grant of the Austrian Science Fund FWF (F3210-N18).

\section{REFERENCES}

1. Trayanova NA, Rice JJ. Cardiac electromechanical models: from cell to organ. Front Physiol. 2011;2:43.

2. Roth BJ. Electrical conductivity values used with the bidomain model of cardiac tissue. IEEE Trans Biomed Eng. 1997;44:326-328.

3. Dobrzynski H, Anderson RH, Atkinson A, et al. Structure, function and clinical relevance of the cardiac conduction system, including the atrioventricular ring and outflow tract tissues. Pharmacol Ther. 2013. in press.

4. Bayer JD, Blake RC, Plank G, Trayanova NA. A novel rule-based algorithm for assigning myocardial fiber orientation to computational heart models. Ann Biomed Eng. 2012;40:2243-2254.

5. Keller DU, Weiss DL, Dossel O, Seemann G. Influence of $\mathrm{I}(\mathrm{Ks})$ heterogeneities on the genesis of the T-wave: a computational evaluation. IEEE Trans Biomed Eng. 2012;59:311322 .

6. Holzapfel GA, Ogden RW. Constitutive modelling of passive myocardium: a structurally based framework for material characterization. Philos Trans A Math Phys Eng Sci. 2009;367:3445-3475.

7. Prassl AJ, Kickinger F, Ahammer H, et al. Automatically generated, anatomically accurate meshes for cardiac electrophysiology problems. IEEE Trans Biomed Eng. 2009;56:1318-1330.

8. Mahajan A, Shiferaw Y, Sato D, et al. A rabbit ventricular action potential model replicating cardiac dynamics at rapid heart rates. Biophys J. 2008;94:392-410.

9. Rice JJ, Wang F, Bers DM, de Tombe PP. Approximate model of cooperative activation and crossbridge cycling in cardiac muscle using ordinary differential equations. Biophys J. 2008;95:2368-2390.

10. Plank G, Liebmann M, Weber dos Santos R, Vigmond EJ, Haase G. Algebraic multigrid preconditioner for the cardiac bidomain model. IEEE Trans Biomed Eng. 2007;54:585-596. 